\title{
El músculo esfenomandibular ¿es un músculo independiente 0 es un fascículo del músculo temporomandibular?
}

Sphenomandibular muscle, is an independent muscle or is a fascicle of temporomandibular muscle?

Sergio Alvarado-Menacho, ${ }^{1, a, b}$, Carlos Arroyo-Pérez ${ }^{1, a b}$, Juana Delgadillo-Avila ${ }^{1, a, c}$, Sylvia CheinVillacampa ${ }^{1, \mathrm{a}, \mathrm{d}}$, Patricia Astupinaro-Capristan ${ }^{1, \mathrm{a}, \mathrm{d}}$, Arnaldo Munive-Degregori ${ }^{1, \mathrm{a}, \mathrm{b}}$, Tulio AbuhadbaHoyos $^{1, a, d}$, Ada Delgado-Yauyo ${ }^{2, a, d}$, Marcelo Canicoba-Sánchez ${ }^{1, e}$, Daniel Alegre-Carhuachin ${ }^{1, e}$.

\section{RESUMEN}

Desde los años 90, odontólogos británicos describieron la presencia del músculo esfenomandibular como un músculo masticador, luego fue estudiado por odontólogos brasileros y bolivianos, aún existe la errónea idea que su presencia no es real y se ha cuestionado que sea un músculo independiente. Objetivo: Demostrar la presencia del músculo esfenomandibular como músculo independiente y su relación con el músculo temporal. Material y métodos: Se siguió un proceso de disección especial sugerido por Campohermoso et al, para verificar al músculo esfenomandibular; se trabajó con siete hemicabezas humanas, Resultados: Todos los especímenes estudiados presentaron el músculo esfenomandibular, comprobando una inserción superior en la parte anterior de la apófisis pterigoides del hueso esfenoide retromaxilar muy cercana a la hendidura esfenoidea próxima al ingreso del nervio motor ocular común, y una inserción inferior en la cresta de la cara interna de la apófisis coronoides de la mandíbula; hay independencia de éste músculo con el músculo témporal. La media del promedio del músculo fue de 44,7 mm de largo, por 6,81 mm de ancho. Conclusiones: No es concluyente la presencia del músculo esfenomandibular, se requiere ampliar estudios en otras áreas como son aspectos embriológicos, fisiológicos, estructurales y clínicos, este estudio servirá como inicio de una línea de investigación analítica de los músculos masticadores.

PALABRAS CLAVE: Anatomía regional; músculos masticadores; sistema estomatognático.

Facultad de Odontología, Universidad Nacional Mayor de San Marcos, Lima, Perú.

Facultad de Odontología, Universidad de San Martín de Porres, Lima, Perú.

Docente

Magister en Estomatología / Odontología

Doctora en Ciencias de la Salud

Cirujano Dentista

Estudiante 


\section{SUMMARY}

Since the early 1990s, British dentists described the presence of the sphenomandibular muscle as a chewing muscle, then it was studied by Brazilian and Bolivian dentists, still exists the erroneous idea that his presence is real and has been questioned as an independent muscle. Objective: To demonstrate the presence of muscle sphenomandibular as independent muscle and its relationship with the temporal muscle. Material and methods: Followed a special dissection process suggested by Campohermoso et al, to verify the sphenomandibular muscle; they worked with seven human hemicabezas. Results: all specimens studied showed muscle sphenomandibular, checking an insertion in the front part of the bone pterygoid process sphenoid retromaxillary very close to the cleft sphenoidal close to the entrance of the common ocular motor nerve, and an insert lower on the crest of the inner side of the jaw coronoid process; independence of this there is muscle with the temporal muscle. The mean average of the muscle was $44.7 \mathrm{~mm}$ long, by $6.81 \mathrm{~mm}$ wide. Conclusions: The presence of the sphenomandibular muscle is not conclusive, is required to further studies in other areas such as embryological, structural, physiological and clinical aspects, this study will serve as the beginning of a line of analytical research of the chewing muscles.

\section{KEY WORDS: Regional; anatomy; masticatory muscles; stomatognathic system.}

\section{INTRODUCCIÓN}

Cuando hablamos de los músculos masticadores y específicamente de los músculos elevadores, nos referimos a los músculos temporal, masetero y pterigoideo interno y se suma en ésta función el pterigoideo externo vientre superior (1-3), también estudiados por elementos finitos (4).

Algunos autores británicos y americanos indicaron que el músculo esfenomandibular, no es un apéndice del músculo temporal, sino que se presenta como un músculo independiente (5), y que es un agonista del pterigoideo lateral (6). Posteriormente fue confirmada su presencia por odontólogos brasileros y presentado en La Paz Bolivia, y posteriormente fue en Sucre el año de 1994 (7). Fue presentado por Tavares y Da Silveira en el IX Congreso Internacional de Odontología del Distrito Federal del Instituto de ensino y pesquisa en medicina y odontología legal (IBEMOL) en Brasilia el año 2001 (8).

Manifiestan los autores que éste músculo no fue descrito anteriormente por la dificultad de la disección de la fosa infratemporal, ya que se tiene que realizar una disección especial para no lesionar a dicho musculo, puesto que, con la disección tradicional, fácilmente puede ser seccionado (7).

Raulino Naves y Marcos Ávila concluyeron que el músculo esfenomandibular es una entidad separada e independiente del músculo temporal con sus fibras de origen no en la parte maxilar del hueso esfenoides, pero si desde el complejo cigomático frontal (9).

Existe gran interés en la ubicación del músculo esfenomandibular (10), ya que se le asocia a sintomatología relacionada con alteración de la visión o dolor ocular con Desordenes Témporomandibulares (DTM) (7). Naves y Avila relacionaron la hiperactividad muscular del músculo esfenomandibular con síntomas visuales concluyendo que siendo el músculo esfenomandibular independiente del músculo temporal y que está situado en las proximidades de la órbita, justifican la hiperactividad de éstos músculos con una asociación significativa en los DTM con manifestación de dolor ocular referido (11).

Naves et al., estudiaron la relación de la hiperactividad de los músculos esfenomandibulares relacionados con la baja visión y concluyeron que fue considerable el índice de hiperactividad de los músculos esfenomandibulares en pacientes con DTM, también encontraron que existió una interrelación de los DTM y sintomatología visual de baja visión (12), otros autores lo asocian a diferentes patologías (13-16).

Evanisi Palomari (17), Geers y otros autores $(18,19)$, cuestionan la independencia del músculo esfenomandibular, indicando que en primer lugar el 
músculo temporal es una sola entidad, esto se basa en la ausencia de fascia muscular entre los paquetes musculares, en segundo lugar que el músculo esfenomandibular mencionado por Dunn et al., (5), corresponde con el paquete medial o profundo del músculo temporal y en tercer lugar, que la presencia de éste paquete denominado como intermediario estimula nueva verificación de la función del musculo temporal. Akita (20), también lo considera así.

Álvarez et al., indican que los resultados de su estudio permiten afirmar que el músculo esfenomandibular es una estructura constante en nuestro medio y separada e independiente del músculo temporal con sus fibras de origen, no en la parte maxilar del hueso esfenoides, sino en la cara externa del ala mayor del esfenoides, en su cara cigomática, inmediatamente detrás de la cresta subtemporal y su presencia en nuestro medio es, probablemente, por el hábito del acullico -chacchar(pues, la mayor parte de los cadáveres diseccionados fueron de personas del área rural andina) (21).

El objetivo de éste trabajo fue determinar la presencia, inserciones y reparos anatómicos cercanos del músculo esfenomandibular como músculo independiente y su relación con el músculo temporal.

\section{MATERIAL Y MÉTODOS}

La investigación fue observacional, transversal y descriptiva, para lo cual se trabajó con cadáveres adultos disponibles en el anfiteatro de Anatomía de la Facultad de Odontología de la Universidad Nacional Mayor de San Marcos en Lima-Perú (UNMSM). Se estudiaron 07 hemicabezas correspondiente a los cadáveres adultos del anfiteatro de la facultad de Odontología de la UNMSM.

La técnica de recolección de datos fue mediante la observación directa de los indicadores de reparos anatómicos de la fosa subtemporal, acorde con las siguientes etapas:

1. Previamente se calibró a los investigadores para la toma de datos. También se verificó el buen estado de los instrumentos a emplear.

2. Se procedió a diseccionar la región de la fosa subtemporal en las cabezas de los cadáveres, acorde con los parámetros descritos por Campohermoso et al., (7), descubriendo en esta la ubicación e inserciones del músculo esfenomandibular y sus relaciones con:

a. El hueso esfenoides

b. El proceso coronoideo del hueso mandibular

c. La parte posterior de la órbita ocular y el ingreso del nervio óptico y establecer la presencia e independencia del músculo esfenomandibular y su relación con los reparos anatómicos.

La disección estuvo a cargo de dos experimentadas profesoras de anatomía humana y amplias conocedoras de la anatomía del sistema estomatognático, ambas miembros de éste grupo de trabajo. También se contó con el apoyo de un personal técnico en el manejo y conservación de los cadáveres. El corte de las estructuras óseas se realizó con un motor eléctrico con discos de carburundum.

3. Se realizó el estudio antropométrico de los músculos disecados con mediciones cuantitativas en $\mathrm{mm}$ y pulgadas, las cuales se llevaron a cabo con un Calibrador tipo Vernier, marca Truper modelo digital standard y milimétrico, de origen mexicano con código 14388; de acero inoxidable, con tolerancia de 0,001 pulgada y 0,01 mm, con capacidad máxima de 6 pulgadas y $150 \mathrm{~mm}$, y capacidad mínima de 0,001 pulgada y $0.01 \mathrm{~mm}$

4. Organización de los datos en figuras, tablas y gráficos

5. Análisis estadístico descriptivo de los resultados, utilizando el programa SPSS versión 24.

6. Elaboración del informe final

La muestra estuvo conformada por siete medias cabezas de cadáveres del anfiteatro de la facultad de odontología de la UNMSM, las cuales fueron disecadas cortando la mandíbula a nivel del cuello del cóndilo, por debajo del proceso coronoideo y en la parte media de la rama ascendente, para poder exponer la fosa infratemporal y no lesionar las posibles inserciones inferiores del músculo esfenomandibular (figura 1), así mismo se secciono el arco zigomático por detrás del hueso malar y en la parte superior de la órbita para poder observar la parte posterior del maxilar superior y ver los reparos anatómicos de las inserciones superiores del músculo esfenomandibular y su relación con la parte posterior del ojo (figura 2). Se realizó el estudio estadístico descriptivo e inferencial mediante el programa estadístico IBM SPSS Statistics 24.0. 
Alvarado-Menacho S, Arroyo-Pérez C, Delgadillo-Ávila J, Chein-Villacampa S, Astupinaro-Capristan P, Munive-Degregori A, Abuhadba-Hoyos T, Delgado-Yauyo A, Canicoba-Sanchez M, Alegre-Carhuachin D.

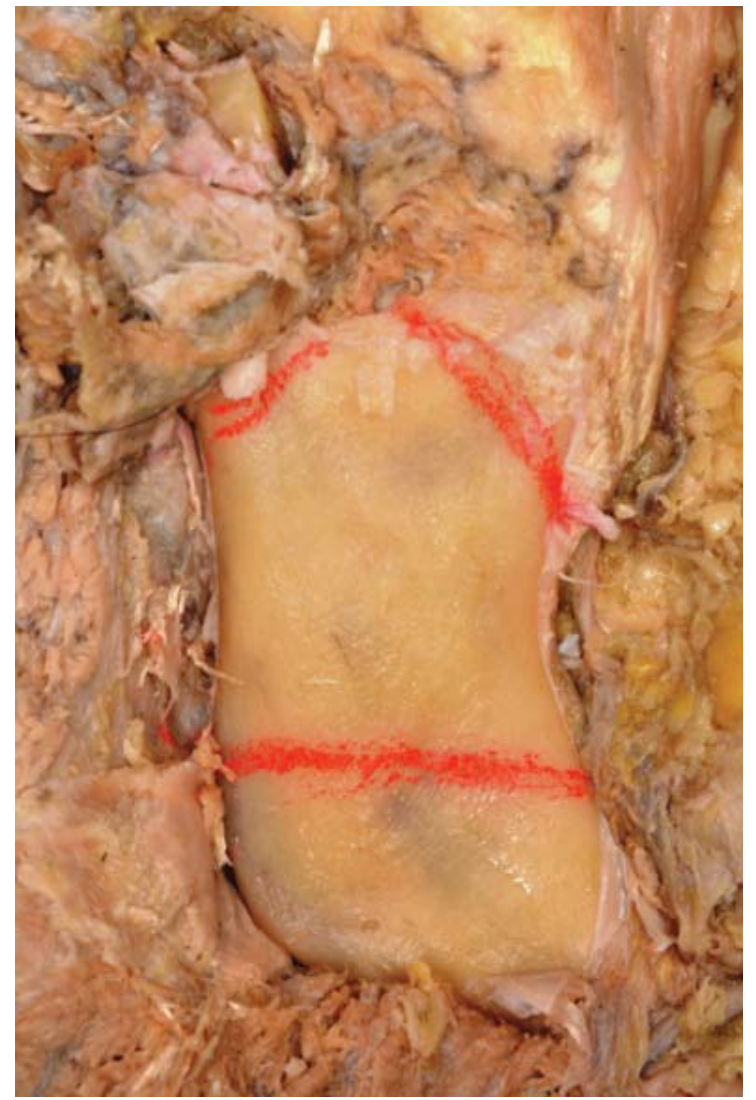

Figura 1. Cortes en la rama ascendente de la mandíbula.

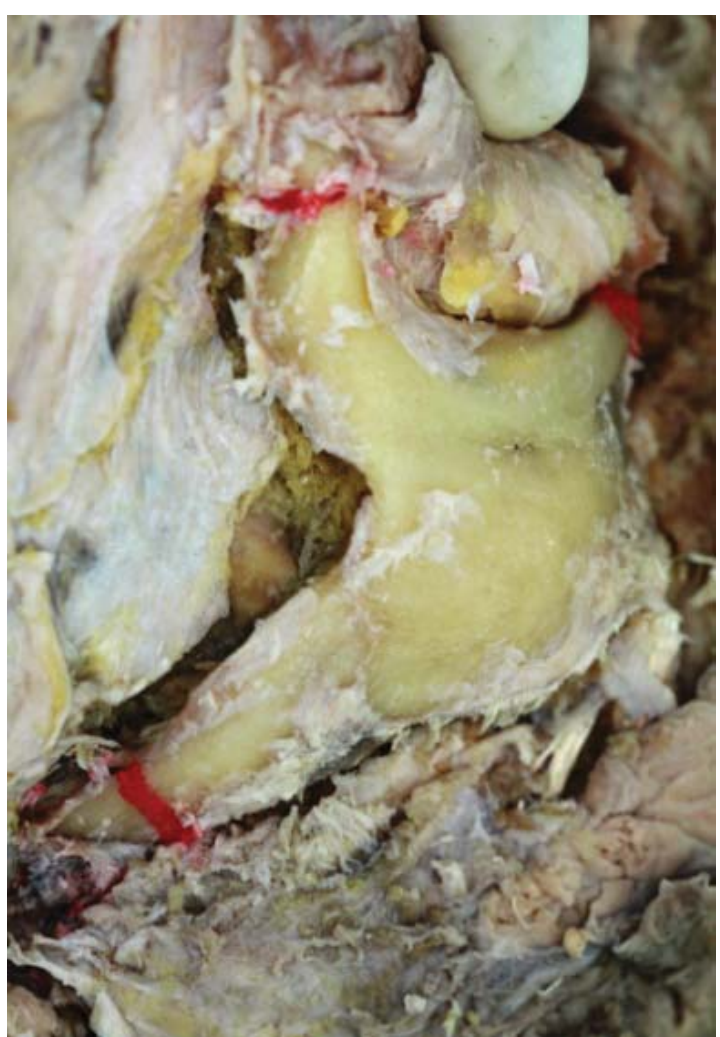

Figura 2. Cortes del arco zigomático y proceso malar.

\section{RESULTADOS}

La inserción superior se encuentra cercana al agujero óptico por donde ingresa el nervio óptico $\left(2^{\mathrm{do}}\right.$ par craneal), nervio encargado de trasmitir información visual al cerebro, pero la inserción del músculo esfenomandibular está mucho más próxima de la hendidura esfenoidal por donde pasa el nervio motor ocular común ( $3^{\text {er }}$ par craneal), nervio también llamado óculomotor ya que es un nervio motor y parasimpático, encargado de inervar los músculos, elevador del párpado superior, recto superior, recto medial, recto inferior y oblicuo inferior, músculos que colectivamente realizan los movimientos oculares, también inervando el esfínter de la pupila (figura 3).

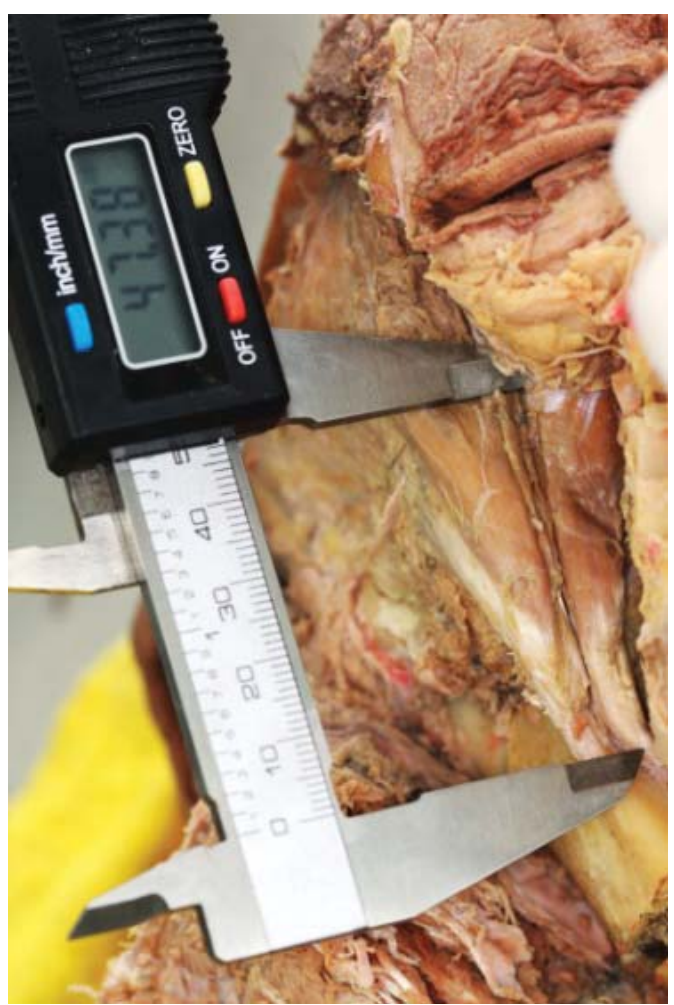

Figura 3. Inserción superior e inferior del músculo esfeno mandibular.

Su inserción de origen se encuentra en la superficie maxilar de la apófisis pterigoides del hueso esfenoides en la parte más anterior y superior del proceso pterigoideo dirigiéndose a la parte superior y posterior de la órbita ocular muy próxima a la hendidura esfenoidal y su inserción inferior se encuentra en la cresta de la cara interna de la apófisis coronoides de la mandíbula (figura 4). 


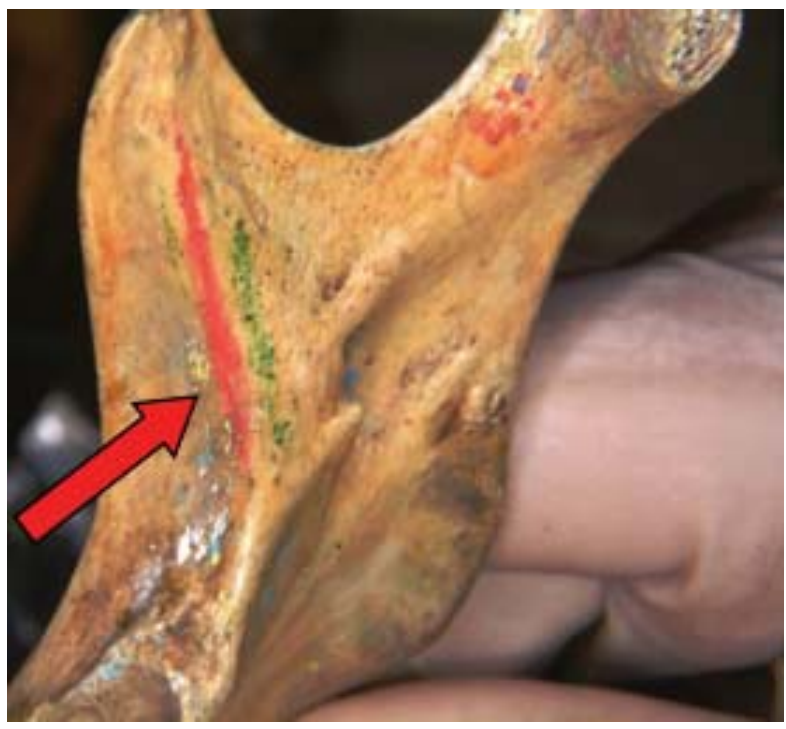

Figura 4. Inserción inferior del músculo esfeno mandibular

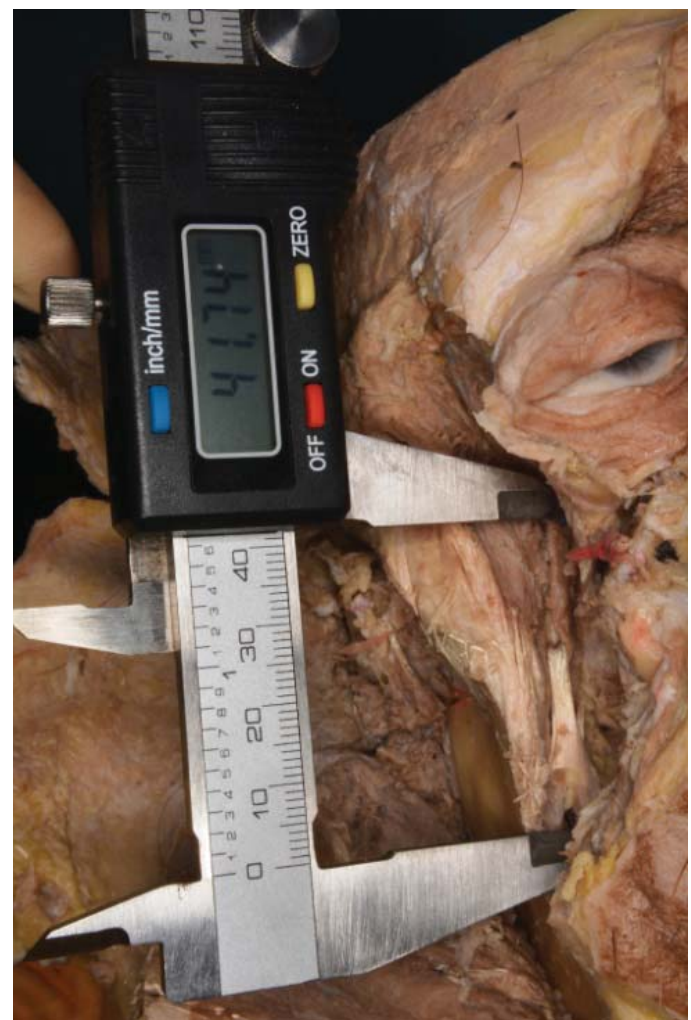

Figura 5. Medición de hemicara derecha.

En las siete hemicabezas disecadas se encontró al músculo esfenomandibular en las inserciones anteriormente descritas, con un promedio de medidas de 44,7 mm de largo (1,759 pulgadas), por 6,81 mm de ancho (0,265 pulgadas), ésta última medida tomada a nivel medio del largo del músculo, el cual se encontró

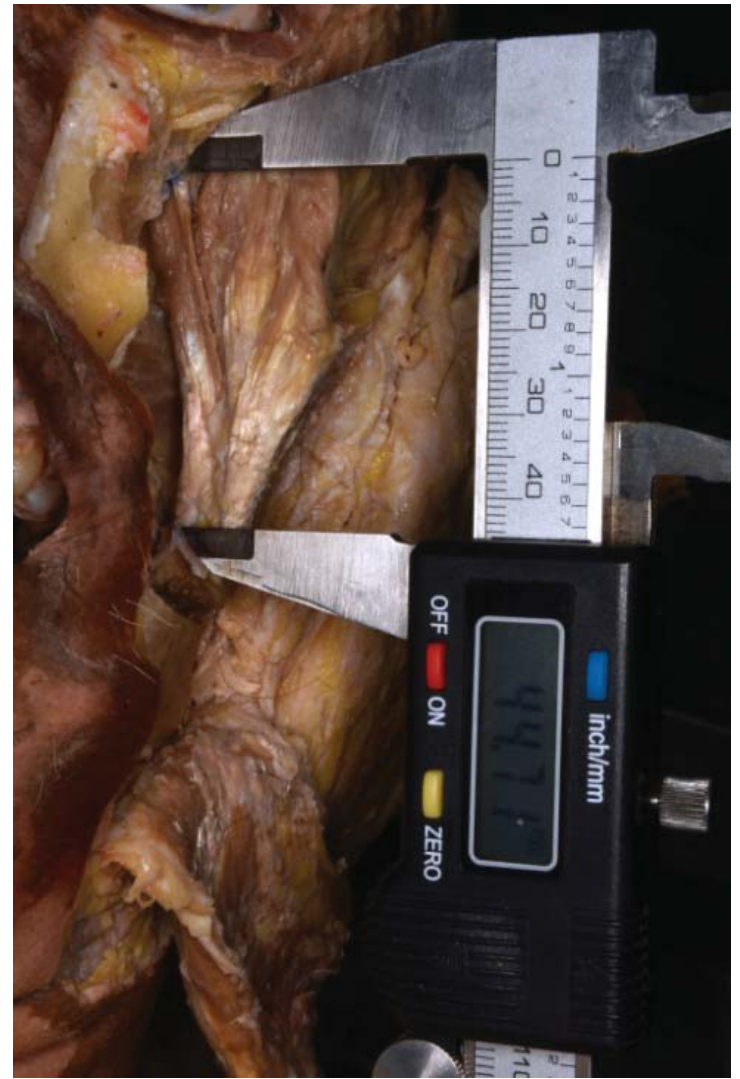

Figura 6. Medición de hemicara izquierda.

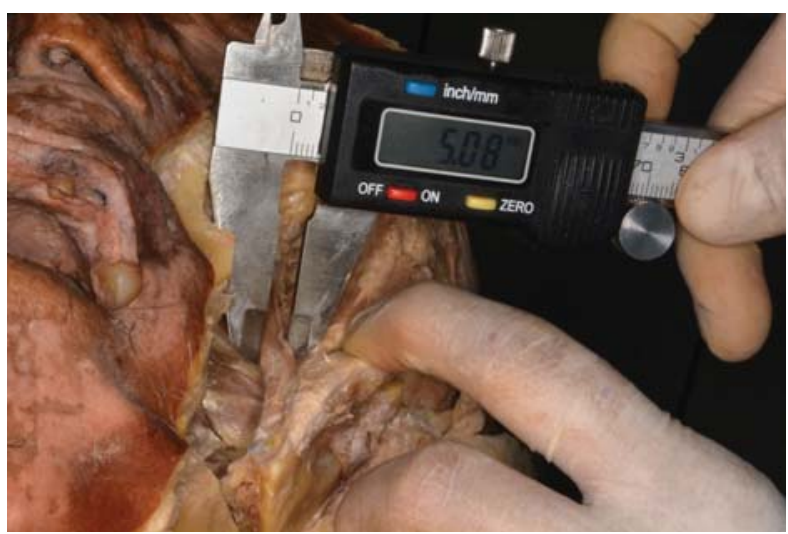

Figura 7. Medición del ancho del músculo esfeno mandibular.

bien diferenciado de la dirección de las fibras internas del temporal insertadas en la parte interna del proceso coronoideo, y de la zona de inserción del pterigoideo lateral (pterigoideo externo), el cual presentaba fibras con dirección horizontal insertadas en el área externa de la apófisis pterigoides del hueso esfenoides (figuras 5, 6 y 7, tabla 1, gráficos 1 y 2). 
Tabla 1. Sexo, Edad aproximada, Lado de las muestras y Medidas en mm y pulgadas del largo y ancho de los músculos esfenos mandibulares disecados.

\begin{tabular}{cccccccc}
\hline Muestra N & Sexo & $\begin{array}{c}\text { Edad en años } \\
\text { (Aprox.) }\end{array}$ & Hemicara & \multicolumn{2}{c}{ mm } & \multicolumn{2}{c}{ Pulgadas } \\
\hline & & & & Largo & Ancho & Largo & Ancho \\
1 & M & 60 & Derecha & 42,61 & 6,29 & 1,678 & 0,247 \\
2 & M & 100 & Derecha & 41,74 & 4,42 & 1,643 & 0,174 \\
3 & M & 100 & Izquierda & 44,71 & 5,08 & 1,76 & 0,2 \\
4 & M & 65 & Derecho & 47,38 & 6,88 & 1,865 & 0,271 \\
5 & M & 58 & Derecho & 36,89 & 5,94 & 1,452 & 0,234 \\
6 & M & 60 & Derecho & 50,91 & 9,24 & 2,004 & 0,363 \\
7 & M & 60 & Izquierda & 48,67 & 9,82 & 1,916 & 0,386 \\
Promedio & & & & 44,7 & 6,81 & 1,759 & 0,265 \\
\hline
\end{tabular}

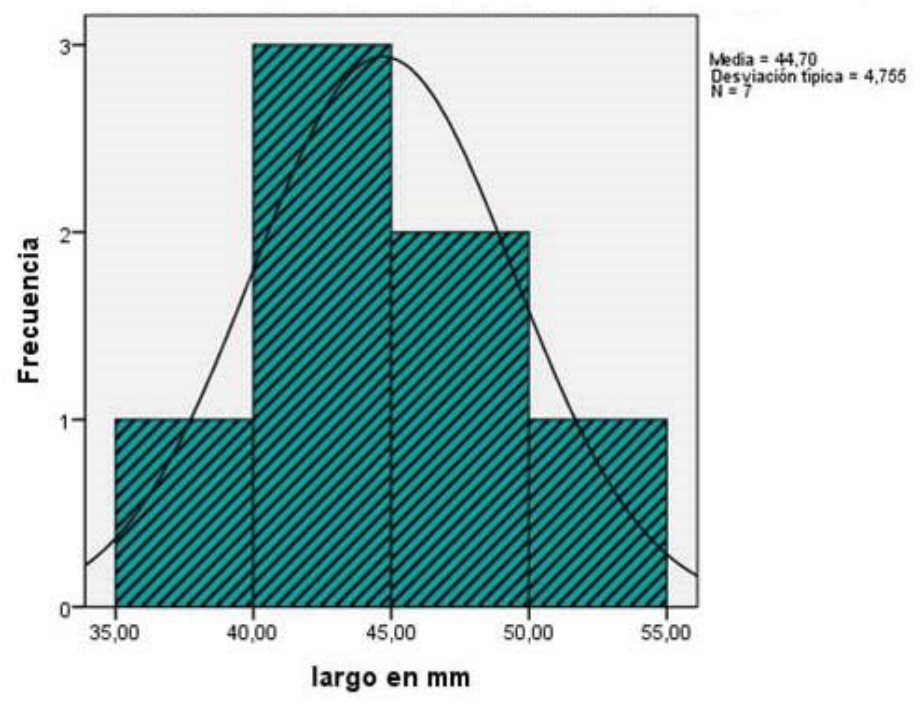

Gráfico 1. Largo del musculo esfenomandibular en mm

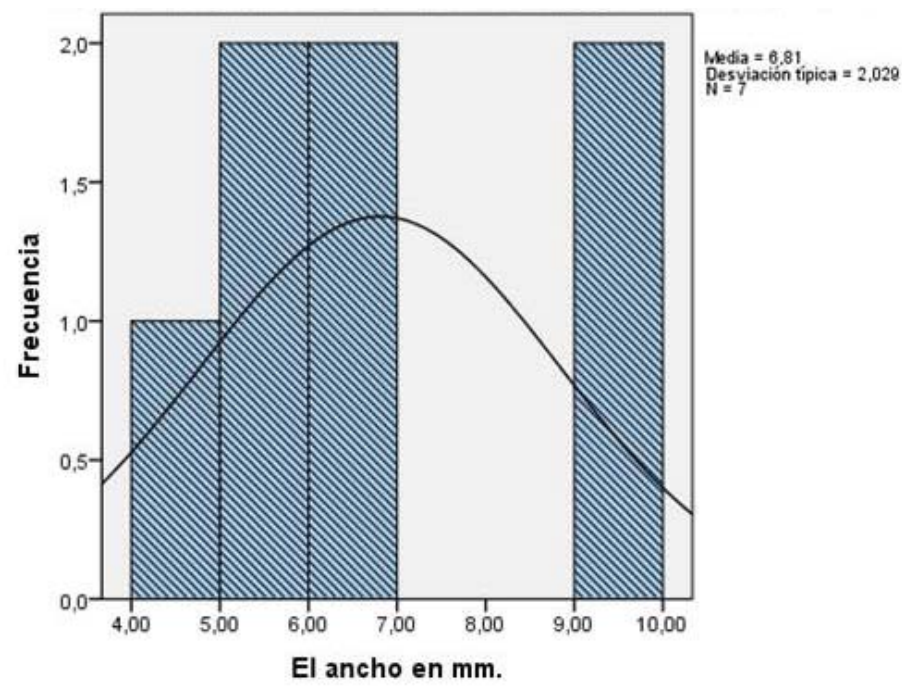

Gráfico 2. Ancho del musculo esfenomandibular en mm 
Se observó la presencia del músculo esfenomandibular en todas las muestras disecadas, cuyas inserciones eran de arriba hacia abajo, de adentro hacia afuera y de adelante hacia atrás, con rangos de medidas de 36,89 - 50,91 mm (1,452 - 2,004 pulgadas), de largo y 4,42 - 9,82 mm (0,174-0,386 pulgadas), de ancho (tabla 1).

\section{DISCUSIÓN}

Generalmente los anatomistas cuando se refieren a los músculos masticadores y específicamente a los músculos elevadores como parte de ellos solamente consideran al masetero, al temporal y al pterigoideo medial $(1,2)$. Otros autores como Okeson, incluyen como músculo sinergista de los músculos masticadores al vientre superior del músculo pterigoideo lateral y por esta razón muchas veces los consideran como cuatro a los músculos elevadores dentro de los músculos masticadores (3), aunque creemos que por un error de redacción, Campohermoso los consideró solamente como masticadores a los elevadores (6); como vemos ninguno de ellos considera la presencia del músculo esfenomandibular como un músculo elevador, a diferencia de nosotros.

A pesar que la muestra es pequeña sugerimos como los autores británicos, que el músculo esfenomandibular, no es un apéndice del músculo temporal, sino que se presenta como un músculo independiente siendo un músculo de reciente descripción desde los años 90. Así mismo suponemos su presencia, así como los odontólogos brasileros que lo presentaron como Campohermoso el año de 1994 (7) y Tavares y Da Silveira en Brasilia en 2001.

Así como lo manifiesta Campohermoso, éste músculo no fue descrito anteriormente por la dificultad de la disección de la fosa infratemporal, ya que se tiene que realizar una disección especial para no lesionar a dicho musculo (7). En nuestros trabajos también pudimos comprobar que la disección no fue sencilla ya que tuvimos que descubrir la fosa infratemporal previamente, seccionando grandes bloques de hueso tanto de la mandíbula como del arco zigomático y del hueso malar con la finalidad de no lesionar las inserciones del músculo esfeno mandibular.

Coincidimos con Raulino Naves y Marcos Ávila ya que ellos en la Universidad de Goias en Brasil, en su estudio topográfico del músculo esfenomandibular, concluyeron que el músculo esfenomandibular es una entidad separada, independiente del músculo temporal, con sus fibras de origen, no en la parte maxilar del hueso esfenoides, pero si desde el complejo cigomático frontal (9). Lo que si pudimos apreciar es que las fibras del músculo esfenomandibular se insertan muy alto en la parte externa y anterior de la apófisis pterigoides del hueso esfenoides y que se encuentra muy cerca a la hendidura esfenoidal y próxima al paso del $3^{\text {er }}$ par craneal, el nervio Motor ocular común.

Según Neyra y Moreira cuando se refieren a la ubicación del músculo esfenomandibular mencionan que presenta hiperactividad en los Desórdenes Temporomandibulares (DTM), y se le asocia a sintomatología relacionada con alteración de la visión o dolor ocular, esto puede ser debido a su proximidad con el nervio óptico ya que la contractura de este músculo supone una compresión con dicho nervio, generando sintomatología asociada a DTMs, situación que desaparece con tratamiento (8). Así mismo Naves y Avila relacionaron la hiperactividad muscular del músculo esfenomandibular con síntomas visuales concluyendo en su estudio que dada la afirmación que el músculo esfenomandibular es independiente del músculo temporal y que está situado en las proximidades de la órbita, justifican la afirmación de que la hiperactividad de éstos músculos tiene una asociación significativa en los DTMs con manifestación de dolor ocular referido (9). Esta afirmación, no fue motivo de nuestro estudio, pero pensamos que tendría más relación con el nervio motor ocular común, que con el nervio óptico ya que la sintomatología dolorosa que se produce en la visión se relaciona más con la hiperactividad muscular que con la sintomatología dolorosa del nervio óptico cuya función es la trasmisión visual, siendo el motor ocular común quien activa todo el aparato motor del ojo, además que la proximidad con la hendidura esfenoidal es más cercana a la inserción superior del músculo esfenomandibular.

En nuestro trabajo no hemos relacionado el dolor con la presencia del musculo esfenomandibular, por eso no podemos comparar nuestro trabajo con el de Naves et al., quienes también estudiaron la relación de la hiperactividad de los músculos esfenomandibulares relacionados con la baja visión y concluyeron que fue considerable el índice de hiperactividad 
de los músculos esfenomandibulares en pacientes con DTMs, también encontraron que existió una interrelación de los DTMs y sintomatología visual de baja visión (12), y otras alteraciones (13-16).

Evanisi Palomari (17), Geers y otros autores $(18,19)$, cuestionan la independencia del músculo esfenomandibular, como lo indican en su estudio titulado ¿¿Músculo Esfenomandibular o Fascículo Profundo del Músculo Temporal? (2013), en el que concluyen que en primer lugar el músculo temporal es una sola entidad, esto se basa en la ausencia de fascia muscular entre los paquetes musculares; en las disecciones realizadas se observó independencia entre las fibras internas del músculo temporal y el músculo esfenomandibular, y la dirección de ambos músculos es completamente diferente; en segundo lugar menciona que el músculo esfenomandibular mencionado por Dunn et al., (5), corresponde con el paquete medial o profundo del músculo temporal relación diferente a la que observamos y en tercer lugar, que la presencia de éste paquete denominado como intermediario estimula nueva verificación de la función del musculo temporal, pensamos que al ser fibras de distinta dirección, la única función en común que tendrían sería la de elevación pero ya que las fibras del músculo esfenomandibular tienen una dirección oblicua, también actuarían como un equilibrador en los movimientos de elevación llevando a la mandíbula a una posición más medial.

Según el trabajo de Orlando Álvarez et al., de la Universidad Mayor de San Andrés en La Paz, Bolivia, realizado en el 2015, nos indica que el músculo esfenomandibular es una estructura constante y separada e independiente del músculo temporal, con lo que coincidimos, y que hay una relación por el hábito del acullico -chacchar- (pues, la mayor parte de los cadáveres diseccionados fueron de personas del área rural andina) (21), no podemos comparar nuestro estudio con sus trabajos, por desconocer el origen de los cadáveres.

Podemos concluir que, a pesar de tener una muestra por conveniencia, no fue suficiente para generalizar la presencia del músculo esfenomandibular, el cual se pudo observar en las disecciones realizadas. Existen muchos otros aspectos además del anatómico que deben ser analizados para afirmar la presencia del músculo esfenomandibular como un músculo independiente, como son, exhaustivos estudios embriológicos, morfológicos, fisiológicos, estructurales además de estudios clínicos para poder relacionarlo con la patología descrita. A pesar de las observaciones realizadas, nos queda la duda de su independencia con el músculo temporal ya que se debe seguir profundizando estudios sobre la relación de ambos músculos. Pensamos que éste trabajo puede ser el inicio de una línea de investigación analítica de los músculos masticadores que sirvan para identificar y relacionar su anatomía y fisiología con alteraciones de los mismos.

Declaración de Financiamiento y de Conflictos de Intereses:Este trabajo de investigación fue auspiciado por el Concejo Superior de Investigaciones del Vicerrectorado de Investigación de la Universidad Nacional Mayor de San Marcos asignándole el código $\mathrm{N}^{\circ} 160501071$

Los autores declaramos no tener conflictos de interés en éste trabajo

Agradecimientos: Agradecemos la colaboración del Sr. Alejandro Vásquez Huamán, técnico anatomista de la Facultad de odontología por su apoyo en la disección de las piezas anatómicas y al Sr. Frank Yauri Bellido, alumno de la facultad de odontología por el apoyo en las tomas fotográficas.

\section{Correspondencia:}

Sergio Alvarado Menacho

Correo electrónico: salvaradom@unmsm.edu.pe

\section{REFERENCIAS BIBLIOGRÁFICAS}

1. Testut L, Latarjet A. Anatomía Humana-Tomo 1. Barcelona: Editorial Salvat; 1977.pp. 782-784.

2. Rouviere H, Delmas A. Anatomía Humana-Tomo 1. Barcelona: Ed Masson SA; 2005. pp.161-168.

3. Okeson J. Tratamiento de oclusión y afecciones temporomandibulares. $7^{\mathrm{a}}$ ed. Barcelona: Elsevier España S.L.; 2013. pp. 14-7.

4. Borie E, Orsi IA, Muñoz G, De Souza LG, Del Sol M. Área de inserción en la mandíbula de los músculos de masticación para ser utilizados en análisis por elementos finitos. Int J Morphol. 2015; 33(4):13771379. doi:10.4067/s0717-95022015000400031

5. Dunn GF, Hack GD, Robinson WL, Koritzer RT, Sharawy M, Tillman LJ. Anatomical observation of a craniomandibular muscle originating from the skull base: The Sphenomandibularis. CRANIO. 1996; 14(2): 97-105. doi:10.1080/08869634.1996.11745955 
6. Fuentes E, Frugone R, Paolinelli C, Hack GD, Bittner V.Electromyographicactivity of the sphenomandibularis and lateral pterygoid human muscles during mandibular lateral movements. Chirurgia. 2012; 25(2):97-9.

7. Campohermoso OF, Bascopé S, Campohermoso O, Torres CC. Músculo esfenomandibular, Quinto masticador. Cuad Hosp Clín. 2009; 54(1):60-3.

8. Neyra D, Moreira R. Estudo anatômico do músculo esfenomandibular e sua relação com estruturas anatômicas vizinhas. Brasilia: Anales del IX Congreso Internacional de Odontología del DF/ Asociación Brasilera de Odontología; 21 al 24 de marzo de 2001. (Fecha de acceso 28 de noviembre de 2015) Disponible en: http://www.ibemol.com.br/ciodf2001/372.asp

9. Naves R, Avila M. Estudio topográfico del músculo esfenomandibular. Stomatos. 2012;18(35):3-8. (Fecha de acceso 28 de noviembre de 2015) Disponible en: http://revodonto.bvsalud.org/scielo.php?script=sci_ arttext\&pid=S1519-44422012000100002\&lng=es\&nr $\mathrm{m}=\mathrm{iso}$

10. Sakaguchi-Kuma T, Hayashi N, Fujishiro H, Yamaguchi K, Shimazaki K, Ono T, Akita K. An anatomic study of the attachments on the condylar process of the mandible: muscle bundles from the temporalis. Surgical and Radiologic Anatomy. 2015; 38(4): 461467. doi:10.1007/s00276-015-1587-4

11. Naves R, Avila M. Estudo da relação entre hiperatividade dos Músculos esfenomandibulares e sintomatología visual. Rev Odontol Bras Central. 2012; 18(35):45-53.

12. Naves R, Melo M, Santos A. Estudo da relação da hiperatividade dos músculos esfenomandibulares e baixa visão. Rev Odontol Bras Central 2012; 21(58):544-7.

13. Wang BH, Moon SJ, Wang H, Olivero WC. (2013). Isolated unilateral temporalis muscle hypertrophy. Journal of Neurosurgery: Pediatrics. 2013; 11(4):451453. doi:10.3171/2013.1.peds12534

14. Fuentes E, Cortés MF, Solari P, Gomez R, Melián A, Suazo G. Tenotomy of the deep portion of the temporalis muscle on trigeminal neuralgia refractory to drugs. Chirurgia. 2015; 28(4):213-6
15. Fuentes-Anabalon E, Llanos-Olmedo S, GómezMaldonado R, et al. Discovery of deep temporalis muscle belly close to maxillary nerve in a patient with trigeminal neuralgia: hypothesis of muscular compression and case report treated by Botox ${ }^{\circledR}$ Onabotulinum toxin tipe-A. Chirurgia. 2016; 29(3):99102.

16. Klein R. Eye disorders in adults. Michigan (MI): Michigan head and neck Institute; 1985. (Citado 26 de junio de 2019) Disponible en: https://www. michiganheadandneck.com/eye-disorders-in-adults/\#. XRSZRvZFzIU

17. Palomari ET, Picosse LR, Tobo MP, Isayama NR, Da Cunha MR. Sphenomandibular muscle or deep bundle of temporal muscle? Int J Morphol. 2013; 31(4):115861. doi:10.4067/s0717-95022013000400002

18. Geers C, Nyssen-Behets C, Cosnard G, Lengelé, B. The deep belly of the temporalis muscle: an anatomical, histological and MRI study. Surgical and Radiologic Anatomy. 2005; 27(3): 184-191. doi:10.1007/s00276004-0306-3

19. Sedlmayr JC, Kirsch CFE, Wisco JJ. The human temporalis muscle: Superficial, deep, and zygomatic parts comprise one structural unit. Clinical Anatomy. 2009; 22(6):655-664. doi:10.1002/ca.20837

20. Akita K, Sakaguchi-Kuma T, Fukino K, Ono T. Masticatory muscles and branches of mandibular nerve: Positional relationships between various muscle bundles and their innervating branches. The Anatomical Record. 2019; 302: 609-619. doi:10.1002/ar.23943

21. Álvarez O, Campohermoso OF, Álvarez G, Campohermoso O. Presencia constante del músculo esfenomandibular (quinto masticador). Cuad Hosp Clín. 2015; 56(1):74-8.

Recibido: 21-02-2019

Aceptado: 17-05-2019 